\title{
Statistical Neutron Emission Model for Neutrino Nuclear Response
}

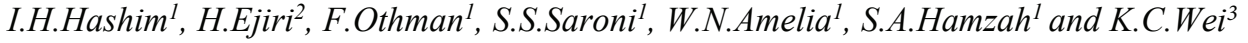 \\ ${ }^{1}$ Dept. of Physics, Faculty of Science, Universiti Teknologi Malaysia (UTM), 81310 Johor Bahru, Malaysia \\ ${ }^{2}$ Research Center of Nuclear Physics (RCNP), Osaka University, Ibaraki-City, 560-0053 Osaka, Japan \\ ${ }^{3}$ Faculty of Nuclear Engineering, Universiti Teknologi Malaysia, 81310 Johor Bahru, Malaysia
}

\begin{abstract}
The studies of neutrino fundamental properties are widely investigated by double beta decay and inverse beta decay. Recent muon capture experiment provides promising ways to directly evaluate the neutrino nuclear responses. This study provides theoretical explanation for neutrino nuclear responses by muon capture experiment. The effects of muon binding energy towards nuclear excitation region and pre-equilibrium (PEQ) and equilibrium (EQ) neutron decay mode will be discussed. The interpretation of muon strength model by comparison of calculator output and recent experimental data may provide relevant information towards determination of nuclear matrix element (NME) and its missing parameter.
\end{abstract}

Keywords: Neutron Emission Model, Neutrino Nuclear Response, Muon Charge Exchange Reaction, Nuclear Matrix Element, Muon Capture Strength.

\section{Introduction}

Theoretical calculations of the weak responses (matrix elements) are challenging since they are sensitive to the nuclear correlations and nuclear medium effects. Thus, experimental work and related theoretical verification are very valuable. Recently nuclear responses of $M\left(\beta^{-}\right)$have been studied extensively by using high-energy resolution $\left({ }^{\beta} H e, t\right)$ experiments at RCNP. On the other hand, observation of $M\left(\beta^{+}\right)$are hard as of no high energy resolution nuclear probes.

It has been pointed out in early 1970s that muon capture reactions (MCR) are alternative to probe the $\beta^{+}$responses [1]. The $\beta^{+}$strengths are obtained by measuring prompt [6] and delayed gamma rays [9-11] from $(\mu, x n)$ reactions with $x$ being the number of the emitted neutrons. So far nuclear gamma rays from MCR were measured to study the nuclear reaction mechanisms $[3,4]$. The prompt gamma rays originating from bound states are excited by $\mu$ capture help the probing of $\beta^{+}$responses at low lying bound states [6,9]. It is hard to extract the $\beta^{+}$strength for individual states.

From the Quasi Random Particle Approximation (QRPA) model, the partial capture rate of low lying excited states contributes to larger error to the weak response of these reactions 
[7,8]. Furthermore, the complicated data analysis of $M\left(\beta^{+}\right)$responses from $\left(t,{ }^{3} \mathrm{He}\right)$ reaction acknowledges that MCR is better candidate in investigating the higher excited states properties. In comparison to QRPA model, this statistical modelling might be able to simulate the process of neutron decay assisting the MCR. It was the first model so study the muon strength distribution contributing to the double beta decay $\beta^{+}$side information [9].

The present study aims to develop the theory to explain the neutrino nuclear response from observation of neutron emission following MCR. The parameters affecting the neutron emission are identified. The neutron spectrum from the neutron emission simulator will be compared with the previous data on muon capture experiment [3]. The relationships of each parameter are identified and express as a function of atomic mass number. These expressions will be implemented to produce the expected isotope mass distribution for muon capture experiment. The $\mu$ capture strength distribution as a function of the excitation energy is derived so as to reproduce the measured reaction branches on the basis of the equilibrium (EQ) and PEQ neutron emission mechanism.

The results of this investigation will shed light on the unknown effective axial-vector coupling constant, $g_{A, e f f}$ and spin iso-spin nuclear parameter, $g_{p}$ which lead to big uncertainties in determining the nuclear matrix element. It will also help the theorists group to decide whether these unknown parameters are suitable with their model or requires adjustment to their parameters.

\section{Theory of Neutron Statistical Model}

A useful model to predict the muon captures strength by implementing the normal distribution for neutron emission events. By using the basis of $\mathrm{C}$ and $\mathrm{C}++$ program, the statistical simulation generates the neutron emission spectrum and compares with the available experimental data. To understand the relationship of neutron emission with the weak nuclear response, the model is constructed into three main parts; the binding energy calculator, neutron emission simulator and isotope population calculator.

The binding energy calculator uses the realistic neutron binding energy and muon binding energy from the raw nuclear mass data reported in nuclear database. This information is important in the neutron emission simulator and isotope population calculator. The fast neutron or pre-equilibrium (PEQ) and slow neutron emission or equilibrium (EQ) are studied by using the neutron emission simulator. The neutron energy spectrum are compared with the previously reported neutron spectrum from muon capture experiment to get the optimize parameters. The combination of best optimize parameter value will be implement in the simulation of isotope population and compare with the experimental data of ${ }^{100} \mathrm{Mo}$ [9].

Fig. 1 illustrates the layout of the present muon charge exchange reaction (MCER). The muon captures on ${ }^{100} \mathrm{Ru}$ excites the nuclei to some bound states at unknown excitation levels. Observation by experiments provides mass distribution from gamma and muonic $\mathrm{x}$-rays related to beta decay process. By studying the mode of neutron decay, the nuclear excitation range at initial bound state after muon captures can be obtained. Comparison between the model isotope population output and the experimental data may help confirm the nuclear excitation region. 


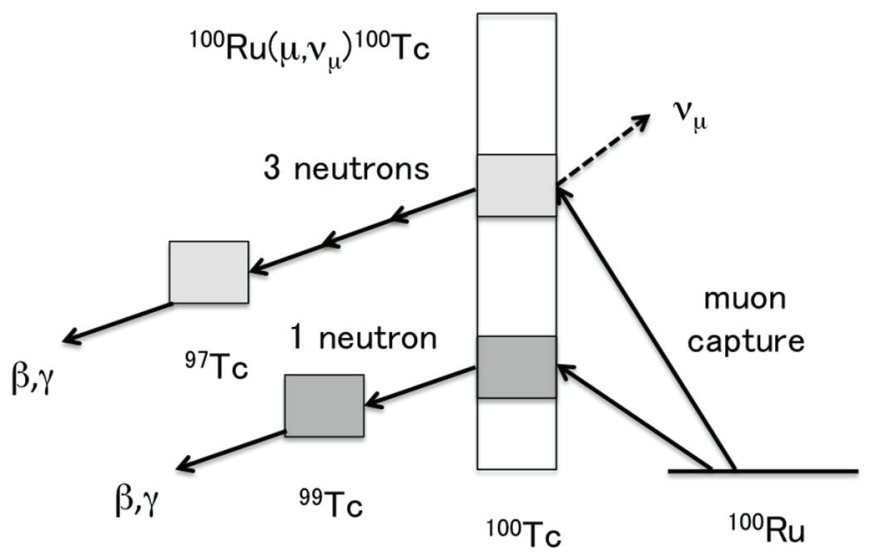

Fig 1: Present MCER ${ }^{100} \operatorname{Ru}\left(\mu, v_{\mu}, \mathrm{xn} \gamma\right)^{100^{-\mathrm{x}}} \mathrm{Tc} v^{-} \tau(\beta)+$ responses

In order to reduce the ambiguity of the calculation, several assumptions are made. The excitation energy, nuclear temperature and atomic mass number often influenced the level density parameter value. Previous studies shown several approximations and models are made for the systematic studies to estimate the accurate value of level density parameter [12]. In this model, we assume that for ${ }^{100} \mathrm{Mo}$ target the level density parameter are set to constant at $14 \mathrm{MeV}$.

The probability of proton emission was expected to be very small where the probability of proton emission begins after three or more neutrons emission. In previous study by [13], shown that most MCR on enriched target shows about $90 \%$ emission from neutron through beta decay. This might cause by the Coulomb force by the proton, which cause higher potential barrier in removing one proton compare to one neutron.

The neutron emission decay mode pertained as challenging issue since it is difficult to differentiate the neutron decay process. In order to study the influence of PEQ neutron emission, the nuclear temperature are set to be in the factor of 4 . The value is assumed from the observation from previously observed neutron emission spectrum. It was expected that this factor are bigger for heavy nuclei.

In muon irradiation experiment, most muon will be captured and some of them will decay. The MCR may excite the nuclei up to $100 \mathrm{MeV}$ due to its high momentum transfer [9][11]. The captured muons are bound to the nuclei at some time interval before muon neutrino releases. The energy to bind the muon with the nuclei are large correspond to the $\mathrm{K}$-shell energy of the atom. Thus, the heavy nuclei possess higher muon binding energy compare to light nuclei. After the bound muon escapes and emitted muon neutrino, the nuclear excitation energy immediately decreases by the factor equals to the muon binding energy. It was expected that at excitation energy of about $21 \mathrm{MeV}$, about two neutrons are emitted. If the excitation energy is greater than the neutron binding energy, more neutron emission are favorable.

The compound and pre-compound nuclei will be formed associates with the emission of EQ and PEQ neutron. The neutron emission spectrum by the Time-of-Flight (TOF) measurement [4][13], shows that the maximum neutron energy are less than $30 \mathrm{MeV}$ and there are small percentage of slow neutrons emitted at higher than $30 \mathrm{MeV}$ range. The ratio of fast neutron to slow neutron are unknown but it was expected to be in the range of $12 \%$ to $30 \%$. By assuming that the probability of neutron emission is equation (1.0) where the first term represent the slow neutron emission which emitted when the nuclear temperature are achieved and the second term represents the fast neutron before achieving nuclear 
temperature. The ratio of neutron emission during EQ and PEQ are studied as a function of atomic mass number, $\mathrm{A}$.

$$
S\left(E_{n}\right)=a \exp \left(-E_{n} / T_{E Q}\right)+b \exp \left(-E_{n} / T_{P E Q}\right)
$$

Where $S\left(E_{n}\right)$ is the neuton emission distribution, $a$ and $b$ are constant and the ratio of $b / a$ should be kept between $15 \%$ to $30 \%$ [9]. The $T_{E Q}$ represents the nuclear temperature at EQ conditions while $T_{P E Q}$ is nuclear temperature at PEQ conditions. The nuclear temperature of slow and fast neutron also differs in some factors as reported in [3]. Since the nuclear temperature factors for ${ }^{100} \mathrm{Mo}$ have not been reported, we use the comparison method to get the optimize value of the factor.

\section{Results and Discussion}

The ratio of $b / a$ from equation (1.0) is manipulated. Three conditions are considered to study their reproducibility towards experimental results. These comparisons might provide inaccurate estimation of neutron energy as well as the neutrino responses. Fig. 2 shows the neutron emission spectrum at $b / a$ ratio of $0.1 \%$ to $1 \%$. For $b / a$ ratio of $0.1 \%$, PEQ neutron emissions are about $30 \%$ to $100 \%$. While the ratio of $0.6 \%$ and $1 \%$, the PEQ neutron emissions increses from $20 \%$ to $70 \%$ and $2 \%$ to $15 \%$ respectively. The values increases as the atomic mass number increases. The higher $b / a$ ratio provides more PEQ neutron emissions.

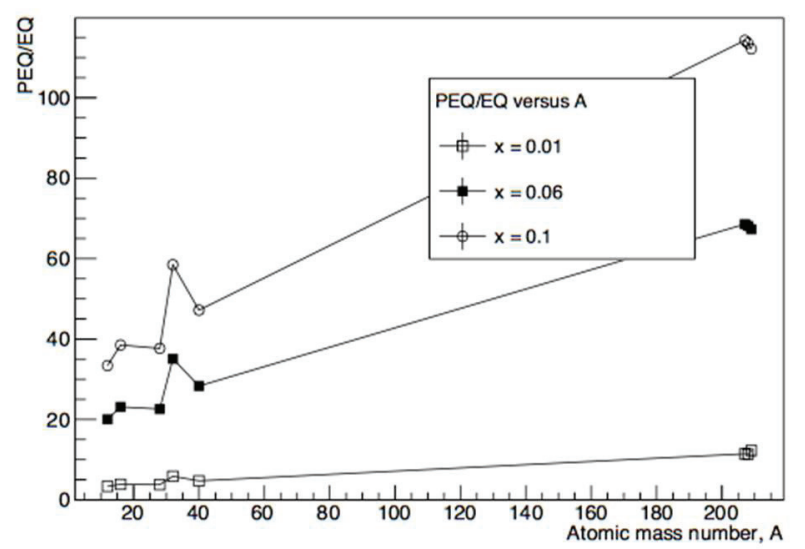

Fig. 2: Ratio of PEQ and EQ neutron emission event at different $b / a$ ratio $(\mathrm{x}=0.01,0.06$ and 0.1$)$

For ${ }^{100} \mathrm{Mo}$ target, the muon binding energy are expected to be $4 \mathrm{MeV}$. If the muon excites the nuclei at $100 \mathrm{MeV}$ and the probabilty of one neutron to emit is $11-14 \mathrm{MeV}$, the maximum excitation energy after the muon neutrino are release at the range of 30 to 40 MeV. Fig. 3 investigate the PEQ neutron emissions when the maximum excitation energy differs. The overall PEQ neutron emissions are in the range of $5 \%$ to $100 \%$ increases as a function of atomic mass number. 


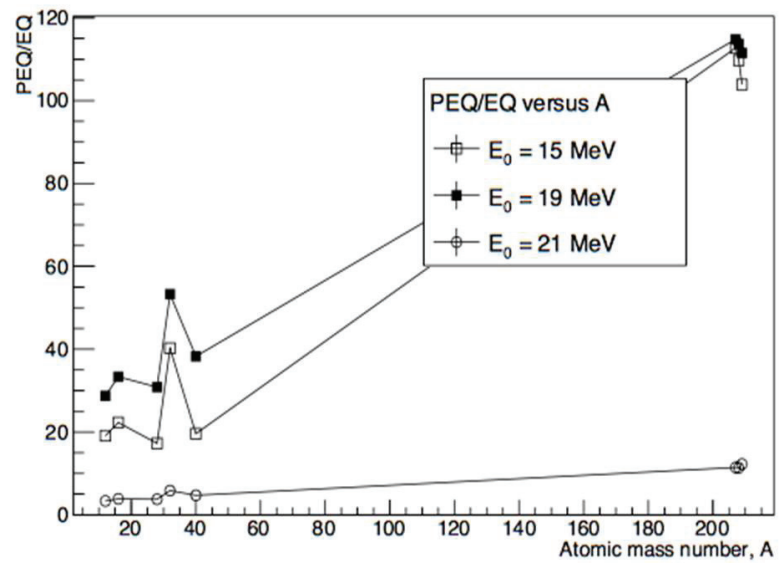

Fig. 3: Ratio of PEQ and EQ neutron emission event at different maximum excitation energy, $E_{0}$

Fig. 4(a) shows the comparison of experimental data by Evseev (1972) [4] with the model using b/a ratio of $0.1 \%$ and excitation energy of $21 \mathrm{MeV}$. The $\chi^{2}$ analysis are used to check which cases are the nearest to experimental data point. All points have very low value of $\Sigma \chi^{2}$, however the combination shows in Fig. 4(a) are the optimize parameters. Since the ${ }^{207} \mathrm{~Pb}$ are heavy nuclei, we would expected that ${ }^{100} \mathrm{Mo}$ shall have almost similar parameters.
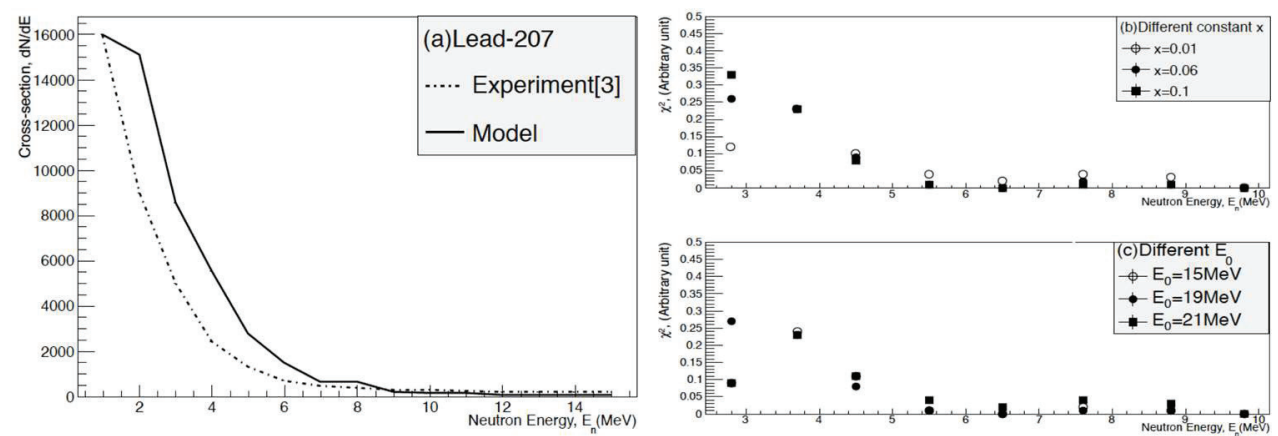

Fig. 4: (a) Comparison for ${ }^{207} \mathrm{~Pb}$ with the model (b) $\chi^{2}$ analysis for different b/a ratio and (c) $\chi^{2}$ analysis for different excitation energy

By using these parameters, the strength distribution of muon capture will be deduced. The muon capture on enriched Mo produces 5 radioisotopes coming from zero to five neutron emission channel [9]. In order to reproduce this experimental data, the capture strength distribution are sampled from 0 to $70 \mathrm{MeV}$ as shown in Fig. 5. The peak strength was appointed in the range of $11 \mathrm{MeV}$ to $14 \mathrm{MeV}$ due to spin-isospin correlation. Most captures at low excitation energy was followed by one neutron emission. A very low population of isotope detected at higher neutron emission. 


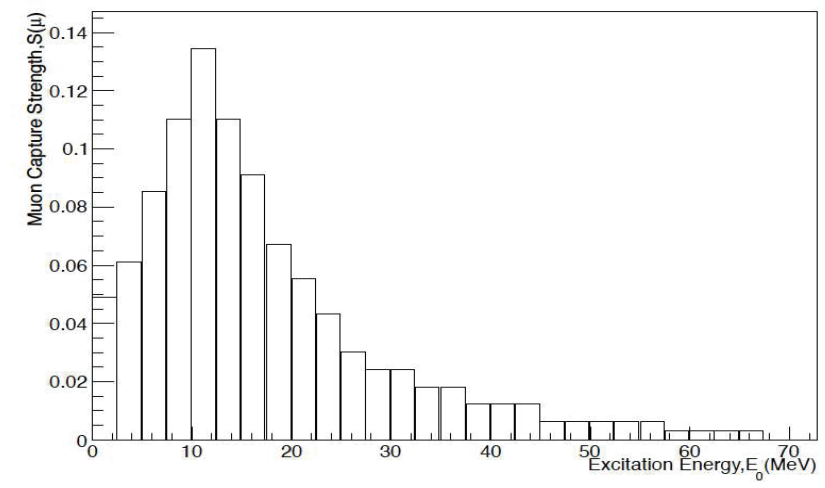

Fig. 5: The capture strength model for comparison with experimental data of ${ }^{100} \mathrm{Mo}$

From Fig. 6, the combination of optimize parameter presents similar observation with the experimental data. Most captures at low excitation energy was followed by 1 neutron emission. A very low population of isotope detected at higher neutron emission. However, there are some point where the experimental observation is two times compare to the model. This only happens at nuclei involved in more than three neutron emission. This is due to the factor of nuclear temperature which are sets to be in the factor of 4 . Systematic study of all the parameters are necessary so that the optimize value for all isotope range can be studied.

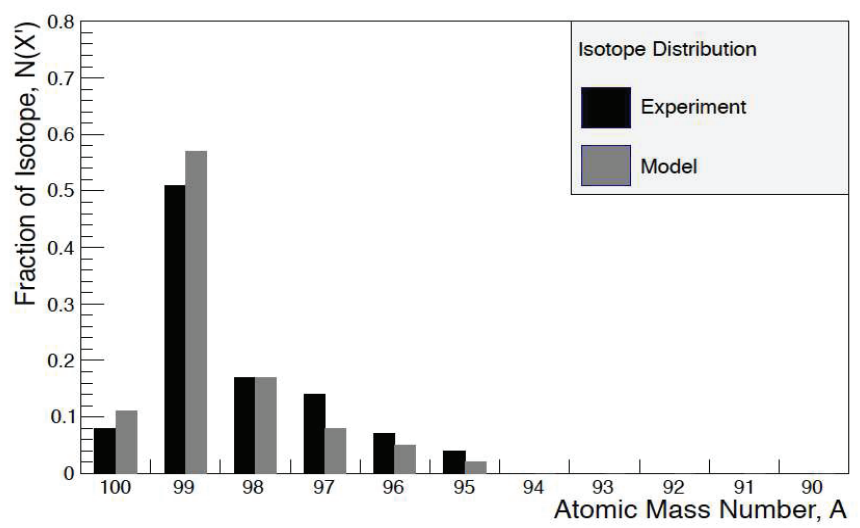

Fig. 6: The isotope population comparison for ${ }^{100} \mathrm{Mo}(\mu, \mathrm{xn} \gamma)^{100-\mathrm{n}} \mathrm{Mo}$

\section{Conclusions}

Muons are a useful probe to study the nuclear structure information that is relevant to $\beta \beta$ decay experiments. It can provide informative discussion on the higher excited states of a nuclei by using weak probe, which are similar to the responses observed in $\beta^{+}$side of $\beta \beta$ decay. The statistical modeling proposed that the initial capture strength accumulates at peak around 11 to $14 \mathrm{MeV}$. The model successfully reproduced the experimental observation using enriched ${ }^{100 \mathrm{Mo}}$ target. The theoretical calculations for the determination of NME by QRPA are necessary for MCR. 
Author would like to express her gratitude to Universiti Teknologi Malaysia Research Grant VOT Q.J130000.2726.11J23 for the funding of this research.

\section{References}

1. H. Ejiri, Proc. EM interactions, Sendai. (1972).

2. H. Ejiri, Phys. Rep. C 338265 and refs. therein (2000).

3. D.F. Measday, Physics Reports 354, 243 and refs. therein (2001).

4. D.F. Measday, et al., Phys. Rev.C 73, 045501. (2006).

5. J. Suhonen, et al., Czech. J. Physics 56 519. (2006).

6. V. Egorov, et al., Czech. J. Phy. 56 523. (2006)

7. H. Ejiri, I.H. Hashim, D. Freckers, private communication. (2014).

8. I.H. Hashim, T.H. Kosmas. private communication. (2014).

9. I.H. Hashim, PhD thesis. Osaka University. (2015).

10. I.H. Hashim, Meson $42^{\text {th }}$ Spring edition. Japan. (2015).

11. H. Ejiri et. al. Journal of Physical Society Japan, 82 044202. (2013).

12. R. Capote. Nuclear Data Sheets 110 3107-3214. (2009).

13. P. Singer. Muon Physics Conference. Colorado State University Fort Collins, Colorado, 39-87. (1971). 